\title{
Monte Carlo simulations of charge transport in organic systems with true off-diagonal disorder
}

Mattias Jakobsson, Mathieu Linares and Sven Stafström

\section{Linköping University Post Print}

N.B.: When citing this work, cite the original article.

Original Publication:

Mattias Jakobsson, Mathieu Linares and Sven Stafström, Monte Carlo simulations of charge transport in organic systems with true off-diagonal disorder, 2012, Journal of Chemical Physics, (137), 11.

http://dx.doi.org/10.1063/1.4748796

Copyright: American Institute of Physics (AIP)

http://www.aip.org/

Postprint available at: Linköping University Electronic Press

http://urn.kb.se/resolve?urn=urn:nbn:se:liu:diva-74319 


\section{AIP Chemical Prysics}

Monte Carlo simulations of charge transport in organic systems with true off-diagonal disorder

Mattias Jakobsson, Mathieu Linares, and Sven Stafström

Citation: J. Chem. Phys. 137, 114901 (2012); doi: 10.1063/1.4748796

View online: http://dx.doi.org/10.1063/1.4748796

View Table of Contents: http://jcp.aip.org/resource/1/JCPSA6/v137/i11

Published by the American Institute of Physics.

Additional information on J. Chem. Phys.

Journal Homepage: http://jcp.aip.org/

Journal Information: http://jcp.aip.org/about/about_the_journal

Top downloads: http://jcp.aip.org/features/most_downloaded

Information for Authors: http://jcp.aip.org/authors

\section{ADVERTISEMENT}

\section{ACCELERATE COMPUTATIONAL CHEMISTRY BY 5X. TRY IT ON A FREE, REMOTELY-HOSTED CLUSTER. \\ NVIDIA.}




\title{
Monte Carlo simulations of charge transport in organic systems with true off-diagonal disorder
}

\author{
Mattias Jakobsson, ${ }^{\text {a) }}$ Mathieu Linares, ${ }^{\text {b) }}$ and Sven Stafström \\ Department of Physics, Chemistry and Biology (IFM), Linköping University, SE-58183 Linköping, Sweden
}

(Received 5 June 2012; accepted 13 August 2012; published online 17 September 2012)

\begin{abstract}
In this work, a novel method to model off-diagonal disorder in organic materials has been developed. The off-diagonal disorder is taken directly from the geometry of the system, which includes both a distance and an orientational dependence on the constituent molecules, and does not rely on a parametric random distribution. The geometry of the system is generated by running molecular dynamics simulations on phenylene-vinylene oligomers packed into boxes. The effect of the kind of randomness generated in this way is then investigated by means of Monte Carlo simulations of the charge transport in these boxes and a comparison is made to the commonly used model of offdiagonal disorder, where only the distance dependence is accounted for. It is shown that this new refined way of treating the disorder has a significant impact on the charge transport, while still being compliant with previously published and confirmed results. () 2012 American Institute of Physics. [http://dx.doi.org/10.1063/1.4748796]
\end{abstract}

\section{INTRODUCTION}

Charge transport in disordered materials has been a widely studied subject ever since the beginning of the solid state electronics era. The subject is still a very active research topic, in particular with the increased interest in low cost electronic materials based on processable conjugated polymers such as poly(p-phenylene vinylene) (PPV). The disordered nature of these polymeric materials makes the exact morphology unknown. Since the microscopic electron transfer rates that control the macroscopic charge transport depend crucially on the morphology, this lack of information remains an obstacle in the search for a full understanding of the electron transport properties.

The theoretical search for transport coefficients is based on the fundamental nature of transport below the mobility edge in disorder materials, namely, that the carriers move by direct tunneling between localized states. In this hopping type of transport, the transition rates between the localized states (or sites) are usually taken from Miller-Abrahams ${ }^{1}$ or Marcus $^{2}$ theory, the latter if polarization and/or geometrical reorganization effects (polaron effects) must be accounted for.

Most studies of hopping transport in conjugated polymeric systems have so far been based on a simplified model focused on the distance between the sites active in the electron hopping process, ${ }^{3}$ even though it is well known that the detailed orientation ${ }^{4-7}$ of localized segments with respect to each other is crucial for the electronic overlap between these sites. The major disadvantage of many of the models used is that the disorder in the system only appears as a diagonal term describing the variations in the potential in the system. This will most certainly limit the validity of the model and make direct comparison with experimental results less reliable.

\footnotetext{
a)Electronic mail: mattias.jakobsson@liu.se.

b) Also at Swedish e-Science Research Center (SERC), SE-100 44 Stockholm, Sweden.
}

In this work, we take the studies of charge transport one step further and introduce the effects of both distance and orientational disorder into the calculations of the transfer rates. In this way, the off-diagonal disorder comes in naturally via the calculation of the transfer integrals between the constituent units active in the transport process.

It is well known that a polymer chain in itself is disordered to an extent that the electronic states are localized to segments, or chromophores, ${ }^{8,9}$ along the chain. We have based our work on such segments of PPV chains and model the system by performing molecular dynamics (MD) simulations on (large) boxes of phenylene-vinylene (PV) oligomers. The distribution of these oligomers within the box constitutes our system and the intermolecular transfer integrals are calculated based on the positions of each individual atom in the system.

The methodology is presented in some detail in Sec. II below; first we describe the MD simulations in Sec. II A followed by the calculation scheme to obtain the diagonal and off-diagonal disorder based on the MD results in Secs. II B and II C. The simulation technique for obtaining the charge transport properties is described briefly in Sec. II D. In the results section we focus mostly on the mobility of the charge carriers and how the mobility and its temperature and electric field dependence are affected by introducing the orientational disorder. Finally, we present a short summary of our results in Sec. IV.

\section{METHOD}

In order to simulate charge transport at the macroscopic level, a structure of the organic material is needed. In this work, this structure was obtained by running MD simulations on four and six monomer long PV oligomers packed into boxes. Three systems were created in this way: (i) a pure 4-PV system, (ii) a pure 6-PV system, and (iii) a mixed 4-PV 

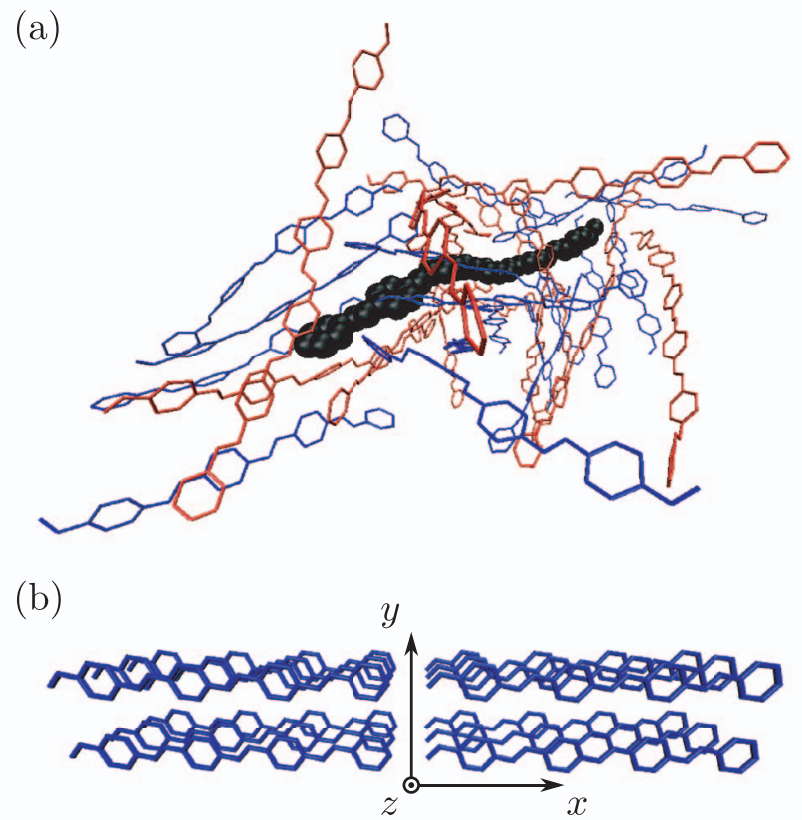

FIG. 1. Part (a) shows a donor oligomer (in black) and its surrounding 4-PV (in blue) and 6-PV (in red) potential acceptor oligomers. Part (b) shows the structure of the model system. The hydrogen atoms of the PV oligomers are not rendered.

and 6-PV system. These systems will henceforth be referred to as the 4-PV, the 6-PV, and the 4/6-PV system, respectively.

The reason we chose to study this type of systems is because of the difficulties of realistically model long chains of polymers at an atomic level. The idea is that the disordered blend of oligomers should capture the chromophore picture ${ }^{8}$ of charge transport in PPV, where the charge carriers make both intra and inter-chain transitions between different segments of the chains. The details of the systems, such as size and ratio, will be given in Sec. II A. A sample from the 4/6-PV system can be seen in Fig. 1(a).

A fourth system was also created. This system, henceforth referred to as the model system, is a simple-cubic molecular crystal of coplanar 4-PV oligomers. The geometry of this system is shown in Fig. 1(b). The orientation was chosen so that the major axes of the oligomers is parallel to the $x$ direction, while the plane of the molecules lie face to face in the $z$ direction. There should hence be a substantial $\pi$-stacking in the $z$ direction (the stacking direction). This system will be discussed further in Sec. II C below.

The charge transport in all four systems is described by the Marcus formula ${ }^{2}$ for the inter-oligomer transition rates,

$$
v_{i j}=\frac{2 \pi}{\hbar}\left|H_{i j}\right|^{2} \frac{1}{\sqrt{4 \pi \lambda k T}} \exp \left[-\frac{\left(\Delta G^{0}+\lambda\right)^{2}}{4 \lambda k T}\right] .
$$

The focus in this article is mainly on the transfer integrals, $H_{i j}$, between the oligomers. This term describes the electronic coupling between a pair of PV oligomers. Fluctuations in this quantity for the transitions between different pairs in the system is the origin of the off-diagonal disorder. The diagonal disorder, which arise from variations in the energy difference in the on-site energy, $\Delta G^{0}$, of the oligomer pairs, will also be discussed. The third important energy in Eq. (1) is the reorganization energy, $\lambda$, which describes the reorganization in the system associated with the charge transfer. In most of the simulations presented below, this energy will be set to $0.3 \mathrm{eV}$, but simulations were also run with a reorganization energy of 0.1 and $0.5 \mathrm{eV}$. The value was varied due to the uncertainty in primarily the solvational part of this quantity. ${ }^{10,11}$

\section{A. Molecular dynamics simulations}

To build the starting configuration for the MD simulations, we use the program PACKMOL, ${ }^{12,13}$ which creates an initial point by packing molecules in defined regions of space by minimizing the short-range repulsive interactions.

As mentioned above, three systems where built: (i) a box of 22500 oligomers of 4-PV; (ii) a box of 6400 oligomers of 6-PV; (iii) a box containing a mix of 7680 oligomers of 4-PV and 5120 oligomers of 6-PV. The ratio of 4 and 6-PV oligomers of the latter box is chosen to get roughly the same total number of monomers in the 4 and 6-PV oligomers. Molecular dynamics simulations in the NPT ensemble were performed during three ns using the OPLS (optimized potentials for liquid simulations) force field ${ }^{14,15}$ and the GROMACS program $^{16-19}$ with periodic boundary conditions (PBC). The temperature was maintained at $300 \mathrm{~K}$ using a Nosé-Hoover thermostat ${ }^{20,21}$ with a coupling constant of 0.2 ps. The pressure was maintained at 1 atm using the Parrinello-Rahman barostat ${ }^{22,23}$ with a coupling constant of $0.2 \mathrm{ps}$. The cut-off distance applied for both electrostatic and van der Waals interactions was $1.5 \mathrm{~nm}$. After equilibration, the density and size of the boxes converged to the values listed in Table I.

Molecular dynamics simulations were performed on those new boxes in the NVT ensemble during five ns using PBC and the same cut-off distances as above. The temperature was once again maintained at $300 \mathrm{~K}$ using a Nosé-Hoover thermostat.

To analyze the results of the MD simulations, various distributions were collected and visualized. A particularly interesting distribution is that of the minimum inter-oligomer distances for the possible inter-molecular transitions. This distribution is shown in Fig. 2 for the 4-PV, 6-PV, and 4/6-PV systems. To discuss this figure, two definitions need to be made: (i) what are the possible transitions and (ii) what is the inter-oligomer distance. The former is the set of oligomer pairs gathered by going through each oligomer in the system and identifying the 28 closest neighboring oligomers. Hence, the number of possible transitions in a system is 28 multiplied by the total number of oligomers. The value of 28 was chosen to be large enough not to limit the charge transport, but small

TABLE I. The size and density of the MD boxes.

\begin{tabular}{lcc}
\hline \hline System & $\begin{array}{c}\text { Size } \\
\left(\mathrm{nm}^{3}\right)\end{array}$ & $\begin{array}{c}\text { Density } \\
\left(\mathrm{kg} \mathrm{m}^{-3}\right)\end{array}$ \\
\hline $4-\mathrm{PV}$ & $24.2 \times 24.2 \times 24.2$ & 1083 \\
6 -PV & $18.4 \times 18.4 \times 18.4$ & 1025 \\
$4 / 6-P V$ & $21.6 \times 21.6 \times 21.6$ & 1052 \\
\hline
\end{tabular}




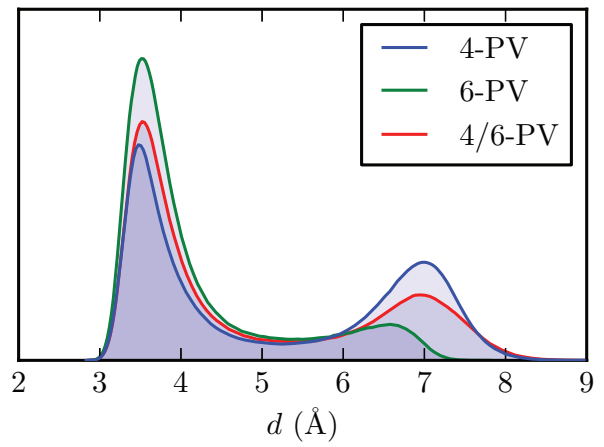

FIG. 2. The distribution of minimum inter-oligomer distances for the possible transitions in the 4-PV, 6-PV, and 4/6-PV systems.

enough to be able to handle computationally. The minimum inter-oligomer distance is defined as the minimum value of the set of distances formed from the distance between all atoms of the first oligomer in a pair to all atoms in the second oligomer of the pair.

Two distinct peaks are clearly visible in the distributions in Fig. 2. The first one, around $3.5 \AA$, is assumed to correspond to the typical nearest neighbor distance, i.e., when the oligomers are packed together, immediately adjacent oligomers do not come closer than about $3.5 \AA$. Outside these nearest neighbors, a second layer of oligomers is visible as another peak. These next-nearest neighbors is located $7 \AA$ away from the original oligomer, i.e., twice the distance of the nearest neighbors. This peak is less pronounced, however, which shows that the system is not strongly aligned, i.e., the major axes of the molecules do not have a preferred direction. If this was the case, the next-nearest neighbor peak should be larger than the nearest-neighbor peak.

The second observation that can be made from Fig. 2 is that long oligomers (6-PV) tend to have more nearest neighbors than the shorter ones (4-PV). This is plausible, since a longer oligomer has a larger surface area, which can fit more neighbors. Once again, this reasoning would break down if the oligomers were aligned, since in that case each neighbor would also occupy more of the surface. Figure 2, hence, makes it very likely that Fig. 1(a) is a representative sample of the disordered MD systems, where the oligomers cross each other at an angle instead of being parallel.

\section{B. Diagonal disorder}

The diagonal disorder, i.e., the DOS of the charge transport orbitals of the oligomers, is generated by calculating the energy of the HOMO for a coplanar oligomer. The coefficients and energy of the orbitals of the 4-PV and 6-PV oligomers where optimized at the RHF/STO-3G level of theory based on the geometries obtained at the same level. A minimal basis set was used in order to have a single $2 p \pi$ coefficient per carbon atom. The use of these coefficients will be explained in Sec. II C.

Once a reference HOMO energy is found, all oligomers are assigned an individual HOMO energy from a Gaussian distribution centered around the reference energy. This is done to model the energetic disorder that arise due to fluctuations

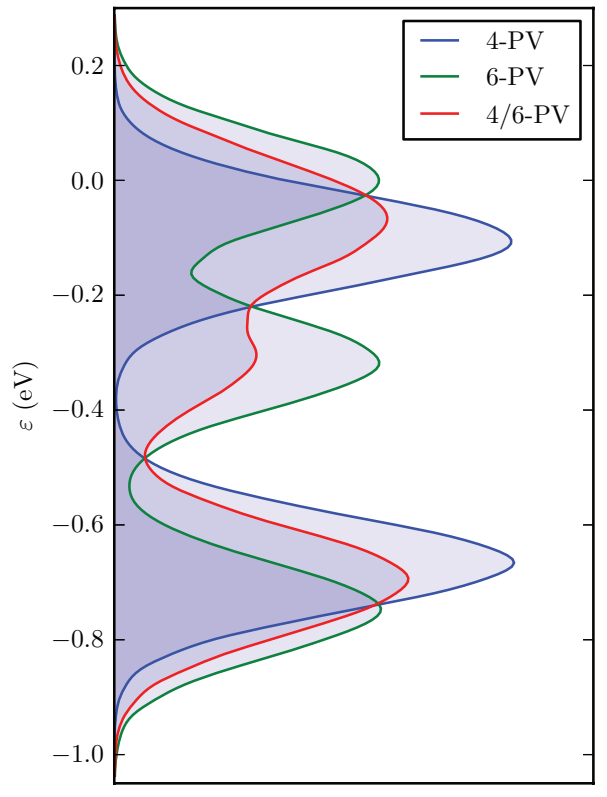

FIG. 3. The diagonal disorder, i.e., the density of states, for the 4-PV, 6-PV, and 4/6-PV systems.

in polarization energy in the system. The standard deviation of the Gaussian distribution is set to $80 \mathrm{meV}$. A value of $90 \mathrm{meV}$ has been reported for PPV, ${ }^{24}$ but a slightly lower value was chosen out of performance reasons for the Monte Carlo simulations.

To make sure that no potential paths for the charge carriers are excluded in the Monte Carlo simulations, this procedure was repeated for the HOMO-1 and HOMO-2 of the oligomers. It should be noted that this is mostly a precaution, the lower lying orbitals are not expected to contribute much to the charge transport since the energy separation from the HOMO level is quite large. The resulting DOS (down to $-1.0 \mathrm{eV}$ ) is shown in Fig. 3 for the 4-PV, 6-PV, and 4/6-PV systems, where the HOMO energy of 6-PV is taken as the reference $(0 \mathrm{eV})$.

For the 4/6-PV system, shown as the red curve in Fig. 3, the DOS is a linear combination of the DOS of the 4-PV and 6-PV systems. This results in a larger diagonal disorder, since two Gaussians with a slight offset in mean value are added together. This is expected to have a negative effect on the mobility in the Monte Carlo simulations.

The diagonal disorder in the model system is the same as in the 4-PV system, although the standard deviation of the Gaussian distributions were chosen to be $50 \mathrm{meV}$ instead of $80 \mathrm{meV}$. This reduction of diagonal disorder should reflect that the fluctuations in polarization energies in this ordered system is smaller than for the disordered MD systems.

\section{Off-diagonal disorder}

The main focus of the method development in this work is to improve the model of the off-diagonal disorder. Previously, the standard method has been to use

$$
H_{i j}=H_{0} \exp \left[-\frac{\beta}{2}\left(r_{i j}-r_{0}\right)\right]
$$


to approximate the electronic transfer integral between two transport molecules. Variations in the inter-molecular distance between the molecules, $r_{i j}$, give rise to fluctuations in the transfer integrals and account for the off-diagonal disorder. The exponential prefactor, $H_{0}$, gives the value of the transfer integral when the molecules are a distance $r_{0}$ apart. The falloff parameter, $\beta$, in Eq. (2) gives the strength of the distance dependence and is related to the localization length, $\alpha$, of the electronic wave function, $\beta=2 / \alpha$.

We have previously used a modified version of Eq. (2) to account for the number of monomer units included in the transport molecules in the case of PPV chromophores. ${ }^{25}$ In this case, the exponential prefactor is given by

$$
H_{0}=\frac{C}{\sqrt{n_{i} n_{j}}},
$$

where $n_{i}$ and $n_{j}$ are the number of monomers in the two participating oligomers. Equation (2) together with this prefactor will be used to compare the traditional distance dependent transfer integrals (henceforth DD transfer integrals) with the new distance and orientational dependent transfer integrals (DOD transfer integrals) introduced in this work.

The major drawback of Eq. (2) is that it fails to take into account the mutual orientation of the molecules. The importance of this orientational dependence was pointed out early by Bässler, ${ }^{3}$ one of the pioneers in the study of hopping charge transport, and attention has been called to it repeatedly since then. ${ }^{4-7}$ However, to the best of our knowledge, the orientational dependence has not yet been used directly in calculations of the mobility. One way to artificially include this dependence is to introduce a disorder in the fall-off parameter, $\beta$, by generating it from a random distribution. The drawback of this method is that the parameters describing this distribution has no physical basis and has to be adjusted to experimental measurements of the mobility.

The lack of an orientational dependence in Eq. (2) also becomes apparent when studying non-spherical molecules. The inter-molecular distance is in this case not well defined and both a center-to-center and an edge-to-edge distance may seem reasonable. As a by-product of our treatment of the electronic transfer integrals, we will show below that the edgeto-edge distance, i.e., the minimum inter-molecular distance, appears to be the most suitable choice.

The new DOD transfer integrals between a pair of oligomers is calculated by taking into account the overlap of each carbon atom in one oligomer with all carbon atoms in the second oligomer. The overlap integral, $S_{i j}$, between two atoms' $2 p \pi$ orbitals, $i$ and $j$, is given by the Mulliken formulas, ${ }^{26}$

$$
\begin{aligned}
S_{i j}\left(r, \theta_{i}, \theta_{j}, \phi\right)= & \cos \theta_{i} \cos \theta_{j} \cos \phi S_{2 p \pi, 2 p \pi}(r) \\
& -\sin \theta_{i} \sin \theta_{j} S_{2 p \sigma, 2 p \sigma}(r) .
\end{aligned}
$$

The three angles $\theta_{i}, \theta_{j}$, and $\phi$ gives the mutual orientation of the two $2 p \pi$ orbitals and $r$ is the inter-atomic distance. This method for calculating overlap integrals has previously been used by, e.g., Hansson and Stafström, ${ }^{27}$ where the definition of the three angles can be found. $S_{2 p \pi, 2 p \pi}(r)$ and $S_{2 p \sigma, 2 p \sigma}(r)$ account for the distance dependence of the $\pi$ and $\sigma$ overlap, respectively, and are given by

$$
\begin{aligned}
S_{2 p \pi, 2 p \pi}(r)= & e^{-r \zeta}\left[1+r \zeta+\frac{2}{5}(r \zeta)^{2}+\frac{1}{15}(r \zeta)^{3}\right], \\
S_{2 p \sigma, 2 p \sigma}(r)= & e^{-r \zeta}\left[-1-r \zeta-\frac{1}{5}(r \zeta)^{2}\right. \\
& \left.+\frac{2}{15}(r \zeta)^{3}+\frac{1}{15}(r \zeta)^{4}\right],
\end{aligned}
$$

where $\zeta$ is a fall-off parameter similar to $\beta$. For the overlap of two carbon atoms, ${ }^{26} \zeta=3.07 \AA^{-1}$.

Once the overlaps between the carbon atoms have been calculated, they are summed up to obtain the resulting molecular overlap integral, ${ }^{28}$

$$
S=\sum_{i j} c_{i} c_{j} S_{i j}
$$

The coefficients, $c_{i}$ and $c_{j}$, are the expansion coefficients for the $2 p \pi$ orbitals $i$ and $j$, respectively, in the wave function describing the molecular orbital. These are obtained from the same Hartree-Fock calculations that gave the molecular orbital energies described in Sec. II B.

Finally, to obtain the molecular transfer integral, we make the approximation that the overlap integral is proportional to the transfer integral,

$$
H=k S \text {. }
$$

The proportionality constant, $k$, is set equal to $-10.6 \mathrm{eV}$, a value obtained from the resonance integrals between pairs of carbon atoms in carbon nanotubes. ${ }^{27}$

The model system in Fig. 1(b) was included to verify the new DOD transfer integrals and the resulting off-diagonal disorder. If the traditional DD transfer integrals are used, the electronic coupling will only depend on the distance between the oligomers in each direction. For oligomers stripped of hydrogen atoms, this distance was chosen to be $3 \AA$ in the $x$ and $y$ direction and $3.5 \AA$ in $z$ direction. Since the center-to-center transition distance is longer in the direction of the major axes and the effect of the electric field is stronger due to this, the DD transfer integrals can be expected to produce the highest mobility in this direction in the model system.

In contrast, if the detailed DOD transfer integrals are used, the electronic coupling is calculated in a much more realistic way. Since the individual overlap between each pair of inter-molecular atomic $2 p \pi$ orbitals are taken into account, this should result in a much stronger electronic coupling in the stacking direction compared to the $x$ - and $y$-direction. Hence, when the DOD transfer integrals are used to model the off-diagonal disorder in the model system, the highest mobility is expected to be observed in the stacking direction. Such behavior is well known and has, for instance, been observed in stacks of PV oligomers ${ }^{29,30}$ and tetrathiofulvalenetetracyanoquinodimethan (TTF-TCNQ) ${ }^{31,32}$ These predictions of the directional dependence of the mobility in the model system are confirmed in Sec. III.

It should be noted that the difference in sign for the expansion coefficients in Eq. (6) is important. For the ordered 
(a)

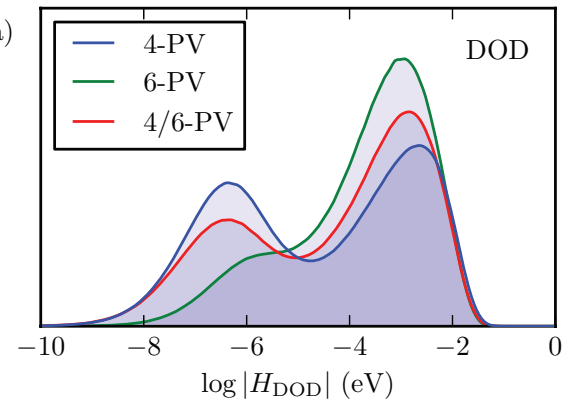

(b)

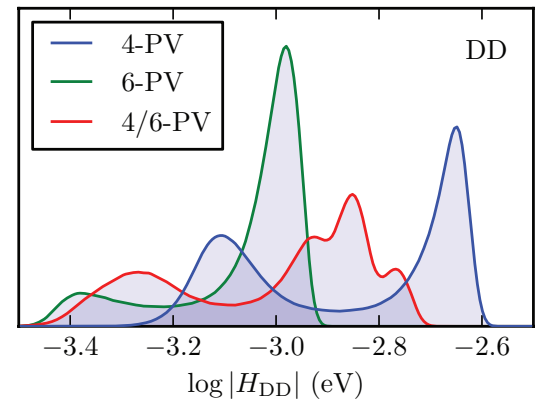

FIG. 4. The off-diagonal disorder, i.e., the distribution of transfer integrals for the possible transitions in the 4-PV, 6-PV, and 4/6-PV systems. Part (a) was generated using the DOD transfer integrals introduced in this work, while part (b) shows the result of using the traditional DD transfer integrals.

model system, the sign of the overlap integrals, $S_{i j}$, that contributes significantly (i.e., are large) to the sum in Eq. (6) all have the same sign. The sign of the coefficients will, hence, determine the sign of the terms in the sum and uniform coefficients results in a larger value of the sum. For the disordered MD systems, the contributing overlap integrals have a more or less random sign and the sign of the coefficients becomes less important when the sum is taken.

Figure 4(a) shows the off-diagonal disorder in the 4-PV, 6-PV, and 4/6-PV systems generated using the DOD transfer integrals described above. Comparing this figure with Fig. 2 makes it very plausible that the peaks observed correspond to the nearest and next-nearest neighbor peaks observed in that figure. In particular, the increase in height of the peaks in Fig. 2 for larger oligomers is observed also in Fig. 4. This results in a larger off-diagonal disorder for the 4-PV system compared to the 6-PV system and the mixed 4/6-PV system lies somewhere in between.

The close correlation between the minimum interoligomer distance and the DOD transfer integrals motivates the use of this distance in Eq. (2). Analogous plots to Fig. 2 of the center-to-center distance exhibit no peaks in the distribution, i.e, the correlation to the transfer integral is much weaker.

A noticeable difference between Figs. 2 and 4(a) is that the peaks in the off-diagonal disorder are broader than the peaks in the distribution of minimum inter-oligomer distances. This is the effect of the orientational dependence that is included in the DOD transfer integrals, i.e., two pairs of oligomers kept at the same distance can produce two different values of the transfer integral depending on the different mutual orientation.
As a comparison, the off-diagonal disorder generated from the DD transfer integrals is shown in Fig. 4(b). For all systems, this is just a transformation of the distribution in Fig. 2 through Eq. (2), since the minimum inter-oligomer distance is used as the variable $r_{i j}$. This is particularly clear for the pure 4-PV and 6-PV systems, since the number of monomers is constant for the oligomers in those systems. For the 4/6-PV system, the possible combinations of the number of monomers in the oligomers involved in the transitions result in a split of the nearest neighbor peak, due to the prefactor in Eq. (3). In principle, this is also true for the next-nearest neighbor peak, but the effect is only visible as a broadening in the figure.

When calculating the DD transfer integrals, the values of $H_{0}$ and $r_{0}$ in Eq. (2) are chosen so that the peaks in the resulting off-diagonal disorder coincide with the peaks in the DOD off-diagonal disorder. The fall-off parameter, $\beta$, is set to $0.6 \AA^{-1}$, which corresponds to a localization length of $3.33 \AA$. This value of the localization length is in the lower range of the interval estimated from experimental measurements. ${ }^{33}$ Comparing the scales of Figs. 4(a) and 4(b) (see also Fig. 7) makes it apparent that this value still results in a much smaller off-diagonal disorder when the DD transfer integrals are used, compared to the DOD transfer integrals.

\section{Monte Carlo simulations}

A brief description of the Monte Carlo method used to simulate the charge transport will be given here, while a more detail description is available in a previous article. ${ }^{25}$ The mobility measured is taken from the steady state velocity of the charge carriers moving in the systems described above. To be able to reach a steady state transport, the systems are made infinite by imposing periodic boundary conditions.

A charge carrier (hole) is inserted in the HOMO of a random oligomer in the system. This oligomer is set as the initial donor oligomer. Next, the transition rates to all 28 acceptor oligomers in the neighborhood of the donor oligomer are calculated. The transition rate to each acceptor oligomer is taken as the sum of the transition rates (Eq. (1)) from the HOMO of the donor oligomer to the three molecular orbitals of the acceptor oligomer, i.e., the HOMO, HOMO-1, and HOMO-2. The transition rates thus calculated is proportional to the transition probability to each of the acceptor oligomers. The actual acceptor is then chosen using a roulette wheel selection method on the acceptor candidates and their corresponding transition probabilities.

Once an acceptor oligomer has been chosen, the dwell time of the charge carrier at the current donor is generated. This dwell time, $\tau$, is drawn from an exponential distribution with a mean value of

$$
\langle\tau\rangle=\left(\sum_{j} v_{i j}\right)^{-1}
$$

where the summation index $j$ runs over all of the acceptor candidates. This dwell time is then added to the current simulation time and the charge carrier is moved from the donor to the acceptor oligomer. Once at the acceptor oligomer, the 
charge carrier is assumed to be in the HOMO orbital, since even if the transition was to the HOMO-1 or HOMO-2 orbital, an intra-molecular transition to the HOMO is expected to happen on a much shorter time scale than the time scale of the next inter-oligomer transition.

During the initial period of the simulations, the charge carriers will be undergoing a relaxation process, where the mean energy of the charge carriers increase (since we study hole transport). This part of the charge transport is not included when calculating the mobility, i.e., the charge carriers are allowed to relax before their velocity is measured in steady state. $^{25}$

The procedure described above is repeated a large number of times until the statistical fluctuations are negligible. Furthermore, the simulations were run on sixteen different snapshots extracted from the last nanosecond of the MD trajectories. The mobility was then taken as the average of the mobility in the boxes at these different times. In this study, the charge transport is assumed to take place in the low charge carrier concentration regime, i.e., there are no interactions between the charge carriers in the simulations.

\section{RESULTS AND DISCUSSION}

The results presented in this section are obtained from Monte Carlo simulations of the charge transport in the systems discussed in the previous section. The variables of the simulations are the applied electric field and the absolute temperature of the system. The quantity not varied will be explicitly given in relation to each figure.

The main points of discussion below will be the difference in the temperature and electric field dependence of the mobility in the different systems generated by MD simulations. In particular, the difference of generating the offdiagonal disorder from DD or DOD transfer integrals will be discussed. Furthermore, the effects of varying the reorganization energy will be investigated along with the difference in mobility in the pure 4-PV and 6-PV systems and the mixed 4/6-PV system. This section starts with a discussion about the model system, however.

Figure 5 shows the temperature dependence of the mobility in the model system for an off-diagonal disorder generated from DD (dashed lines) and DOD (solid lines) transfer integrals. For all curves, the magnitude of the applied electric field is $1 \times 10^{5} \mathrm{~V} / \mathrm{cm}$. The blue circles correspond to an electric field applied in the direction of the major axes of the oligomers. The red diamonds correspond to a field applied in the stacking direction, while the green triangles correspond to a field applied in the $y$ direction of Fig. 1. In this direction, the long edge of the plane of the oligomers face each other and this direction will be referred to as the long-edge direction. All lines show the least squares fit of the Bässler temperature dependence,

$$
\mu(T)=\mu_{1} \exp \left[-\left(\frac{T_{1}}{T}\right)^{2}\right],
$$

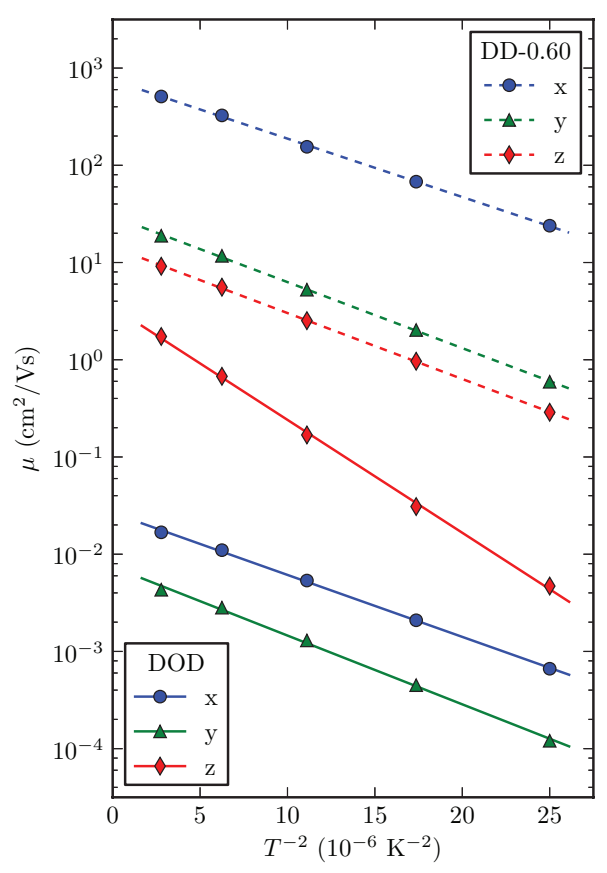

FIG. 5. The mobility as a function of the absolute temperature in the model system. The electric field is $1 \times 10^{5} \mathrm{~V} / \mathrm{cm}$ applied in the $x, y$, and $z$ direction (see Fig. 1(b)) for the blue circles, green triangles, and red diamonds, respectively. The top three dashed curves correspond to DD transfer integrals and the bottom three solid curves correspond to DOD transfer integrals.

to the data points, which is the standard temperature dependence observed for hopping charge transport in a Gaussian DOS. ${ }^{3}$

As predicted and discussed in the introduction to Sec. II, the DD transfer integrals produce the highest mobility for an electric field applied in direction of the major axes of the oligomers, since the orientational dependence is not included. The long-edge direction has a much lower mobility and the stacking direction has slightly lower mobility still, due to the longer inter-oligomer distance in this direction.

The solid curves in Fig. 5 show that the DOD transfer integrals instead produce the highest mobility for the electric field applied in the stacking direction. This is physically motivated and a good validation of our model. Both the direction of the major axes and the long-edge direction are associated with a much lower electronic coupling and hence the mobility is lower. Of these two low mobility directions, the greater influence of the electric field in the direction of the major axes makes the mobility higher for a field in this direction relative to the long-edge direction. This seems reasonable, even though the electronic coupling should be larger in the longedge direction.

Simulations were also performed while varying the direction of the applied electric field on the disordered systems obtained from the MD simulations and the data was used to produce plots analogous to Fig. 5. It was observed that the resulting mobilities in these more realistic systems are very close to isotropic, with less than $30 \%$ relative difference in the magnitude of the mobility in different directions (this should be compared to the more than $30000 \%$ relative difference in Fig. 5). The observed difference was reproducible, however, and cannot be attributed to statistical deviations. This 

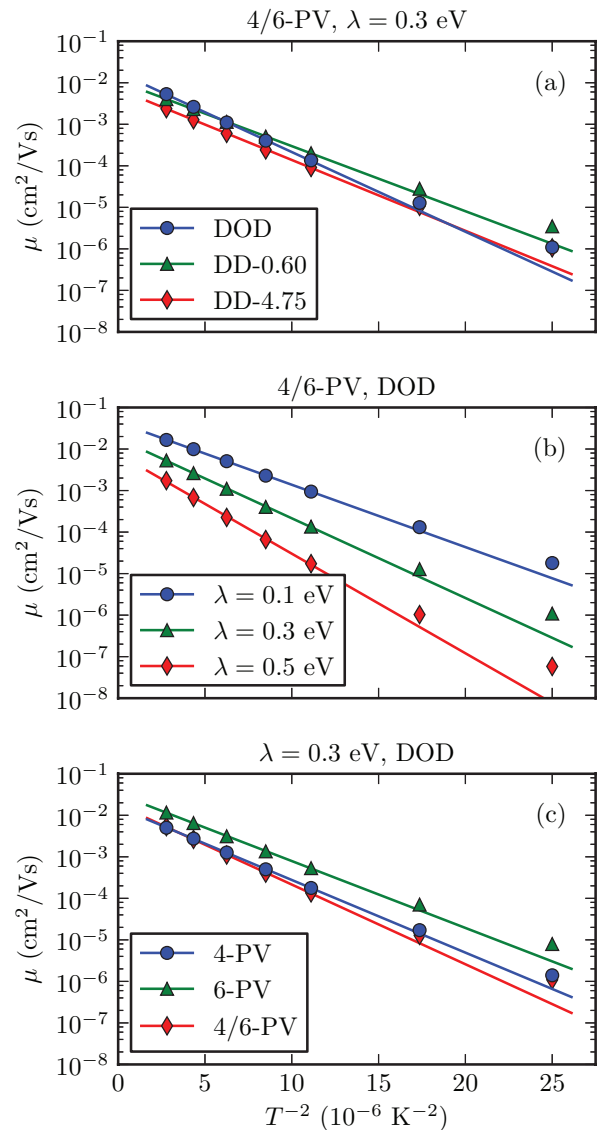

FIG. 6. The mobility as a function of the absolute temperature for (a) different transfer integrals, (b) different reorganization energies, and (c) in different MD systems. The strength of the applied electric field is $1 \times 10^{5} \mathrm{~V} / \mathrm{cm}$.

indicates that there is a slight tendency for the oligomers to line up in a preferred direction, although this tendency is small and could be a local effect that would disappear by running MD simulations on larger boxes. It should also be noted that the observed difference was uniform over a varying magnitude of the applied electric field and the temperature, i.e., the direction of the applied electric field only affect the field and temperature dependence by a constant factor.

Figures 6(a)-6(c) shows the temperature dependence of the mobility for different off-diagonal disorders, reorganization energies, and systems, respectively. The two quantities not varied are noted above the plots. The lines show the least squares fit of the Bässler temperature dependence (Eq. (9)) to the data points corresponding to the five highest temperatures. The two remaining data points at a temperature of 200 and $240 \mathrm{~K}$ diverge from the Bässler dependence and this is most likely due to the finite number of transport sites (oligomers) in the MD systems. This conclusion is drawn because of the fact that the equilibrium energy, $\left\langle\varepsilon_{\infty}\right\rangle$, after the charge carriers are allowed to relax in these systems is lower than that predicted for a Gaussian DOS, ${ }^{3}$

$$
\left\langle\varepsilon_{\infty}\right\rangle=\frac{\sigma^{2}}{k T},
$$

where $\sigma$ is the standard deviation of the Gaussian distribution. The number of oligomers are simply not large enough to ac- count for the rare tail states that dominate the charge transport at these low temperatures.

The blue circles in Fig. 6(a) show the mobility simulated for DOD transfer integrals and the green markers shows the mobility for DD transfer integrals with the fall-off parameter $\beta=0.6 \AA^{-1}$. While Eq. (9) describes both data sets well, the characteristic temperature, $T_{1}$, is $728 \mathrm{~K}$ for the DOD transfer integrals and $614 \mathrm{~K}$ for the DD, which is visible as a difference in slope of the lines fitted to the data sets.

In Fig. 4, it was shown that both the DD and DOD transfer integrals result in an off-diagonal disorder with two distinct peaks. As discussed in relation to that figure, these peaks are assumed to correspond to the typical nearest and nextnearest neighbor distance. It would be interesting to see if the orientational dependence of the DOD transfer integrals can be simulated by increasing $\beta$ in Eq. (2) to make the two peaks for the DD and the DOD off-diagonal disorder coincide. To clarify this, the off-diagonal disorder generated by DOD transfer integrals (blue curve) and DD transfer integrals with $\beta=4.75$ $\AA^{-1}$ (red curve) are compared in Fig. 7. The off-diagonal disorder generated by DD transfer integrals with $\beta=0.6 \AA^{-1}$ is also included in the plot as a reference (green curve).

Increasing $\beta$ increases the characteristic temperature to $657 \mathrm{~K}$ in the temperature dependence of the mobility for the DD transfer integrals. This is closer to the DOD mobility, but not enough to reach a satisfactory agreement. This might be expected, since the off-diagonal disorders shown by the blue and red curve in Fig. 7 differ quite a lot. It can be concluded that the difference between the DD and DOD off-diagonal disorder cannot be compensated for in a straight-forward way by adjusting the parameters.

The blue circles, green triangles, and red diamonds in Fig. 6(b) correspond to increasing values of the reorganization energy, $\lambda$, in the 4/6-PV system. It is obvious that increasing the reorganization energy increases the slope, i.e., the strength, of the temperature dependence. By performing Levenburg-Marquardt least squares fits to the general form of the temperature dependence,

$$
\mu(T)=\mu_{0} \exp \left[-\left(\frac{T_{0}}{T}\right)^{p}\right]
$$

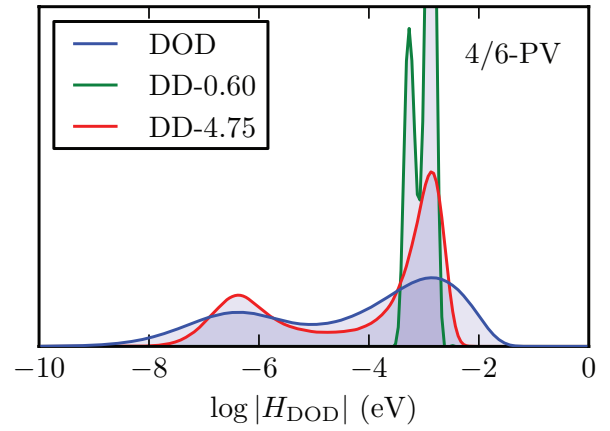

FIG. 7. The off-diagonal disorder in the 4/6-PV system. The red curve shows the off-diagonal disorder generated by DD transfer integrals with $\beta$ $=4.75 \AA^{-1}$. This results in an off-diagonal disorder more similar to the DOD off-diagonal disorder (blue curve). The DD off-diagonal disorder with $\beta$ $=0.6 \AA^{-1}$ is included as a reference (green curve). 
TABLE II. Parameters to fit Eq. (11) to the data points in Fig. 6(b).

\begin{tabular}{lccc}
\hline \hline$\lambda(\mathrm{eV})$ & $\mu_{0}\left(\mathrm{~cm}^{2} / \mathrm{Vs}\right)$ & $T_{0}(\mathrm{~K})$ & $p(-)$ \\
\hline 0.1 & $4.21 \times 10^{-2}$ & 578 & 2.04 \\
0.3 & $2.24 \times 10^{-2}$ & 728 & 1.84 \\
0.5 & $1.41 \times 10^{-2}$ & 932 & 1.68 \\
\hline \hline
\end{tabular}

the values in Table II are recovered. This table shows that not only the slope is affected by the reorganization energy, but also the exponent, $p$, inside the exponential function in Eq. (11). As the reorganization energy increases, the temperature dependence shifts from a more Bässler-like dependence towards a more Arrhenius-like. This trend is observed in all systems studied and for all types of off-diagonal disorder.

The bottom subplot of Fig. 6 shows the temperature dependence of the mobility for the DOD transfer integrals in the 4-PV, 6-PV, and 4/6-PV systems. The 6-PV system has the highest mobility of the three systems, which can be explained by the fact that this system is more ordered compared to the other two (see Figs. 3 and 4). The 4-PV system has a greater amount of off-diagonal disorder, due to the smaller number of nearest neighbors (Fig. 2), and the mixed 4/6-PV system has a greater amount of diagonal disorder, due to the difference in energy level between the HOMO of the 4-PV and 6-PV oligomers. According to Fig. 6(c), these two increases in disorder seem to have roughly the same negative impact on the mobility, both in absolute value and in the slope of the temperature dependence. As will be discussed below, however, this is dependent on the strength of the applied electric field.

Figure 8 shows the applied electric field dependence of the mobility at a temperature of $300 \mathrm{~K}$ and the different subplots are analogous to those in Fig. 6. In subplot (a), the offdiagonal disorder generated with the DOD transfer integrals results in a weaker electric field dependence compared to the DD transfer integrals. This can be explained by the greater amount of off-diagonal disorder associated with the DOD transfer integrals. In a system with large fluctuations in the electronic couplings, the paths that a stronger electric field would open up in a less (off-diagonally) disordered system may be blocked by low transfer integrals. As for the temperature dependence, adjusting the $\beta$ parameter does not seem to be able to bridge the gap between the DD and DOD transfer integrals, as an increase from 0.6 to $4.75 \AA^{-1}$ does not affect the electric field dependence except by a constant shift down in mobility.

Excluding the absolute magnitude of the mobility, Fig. 8(b) shows that the reorganization energy, $\lambda$, has a remarkably small impact on the electric field dependence. The constant drop in mobility, however, is almost two orders of magnitude as the reorganization energy is increased from 0.1 to $0.5 \mathrm{eV}$. Even for the low value of $\lambda=0.1 \mathrm{eV}$, the Marcus inverted region, where the velocity of the charge carriers decrease with increasing field strength, is not visible in the field dependence. It is worth noting, however, that for the traditional DD transfer integrals, this effect starts to become visible for $\lambda=0.1 \mathrm{eV}$ and field strengths above $1 \times 10^{6} \mathrm{~V} / \mathrm{cm}$.
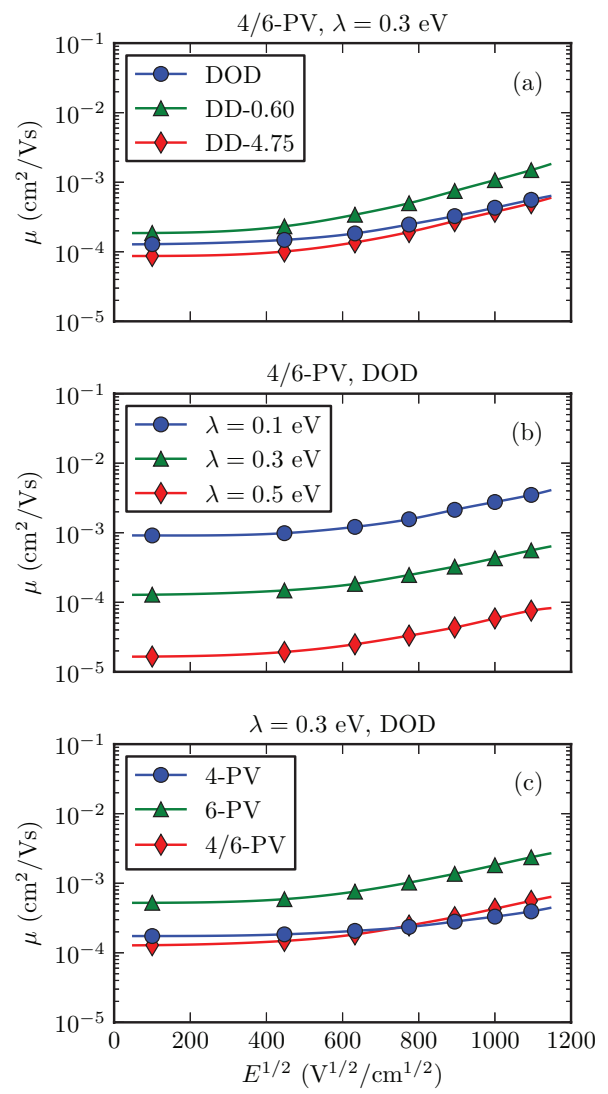

FIG. 8. The mobility as a function of the applied electric field for (a) different transfer integrals, (b) different reorganization energies, and (c) different MD systems. The temperature is $300 \mathrm{~K}$.

Finally, Fig. 8(c) shows the electric field dependence of the mobility in the different MD systems. The relative order of mobility magnitude between the systems was discussed in relation to Fig. 6(c). Here, however, it can be seen that the mixed 4/6-PV system experience a higher mobility than the 4-PV system for high field strengths. This indicates that the amount of diagonal disorder, large in the 4/6-PV system, becomes less important at high field strengths compared to the off-diagonal disorder, which is large in the 4-PV system.

\section{SUMMARY}

We have introduced a novel method to model the offdiagonal disorder in organic systems. By taking into account the full atomic structure of the carbon atoms in the transport molecules and using this to turn the atomic overlap integrals into a molecular transfer integral, the off-diagonal disorder is obtained without the need for a parametric random distribution. This is a major step forward in the modeling of the off-diagonal disorder.

We have showed that for $\pi$-stacked systems, the correct anisotropy comes automatically with this model. Furthermore, charge transport in more disordered systems has been modeled by running MD simulations on 4-PV and 6-PV oligomers packed into boxes. In the studies of these MD systems, the observed temperature and electric field dependence is recognized from previously published theoretical and experimental result of charge transport in a Gaussian DOS. ${ }^{3,36}$ 
A comparison with results based on models with only distance dependent transfer integrals shows that the general form of the temperature dependence, given by Eq. (9), remains the same, but the characteristic temperature, $T_{1}$, is notably higher. The electric field dependence is also sensitive to the different models. In general, the orientational dependent transfer integrals introduced in this work result in a weaker electric field dependence.

The aim of this work is to introduce a direct coupling between a realistic description of the structural disorder and the charge transport in conjugated polymers, PPV in particular. It is not perfectly clear how well this model reproduces experimental data. There are very few experimental results on unsubstituted PPV. A time-of-flight study by Lebedev et al. ${ }^{37}$ on unsubstituted PPV showed an Arrhenius temperature dependence, $\ln \mu \propto T^{-1}$, of the mobility, while we exhibit a temperature dependence somewhere in between the Arrhenius dependence and the Bässler dependence (Eq. (9)) depending on the reorganization energy. Unfortunately, the measurements were made over a rather narrow range of temperatures $(\sim 50 \mathrm{~K})$ and it is difficult to separate the two behaviors. Mobility measurements in PPV derivatives are more abundant. Many of these ${ }^{24,34,35}$ point to the difficulties of making a distinction between the two dependencies. It is, hence, still unclear how to describe the temperature dependence in PPV.

The electric field dependence is usually found to be of a Poole-Frenkel type, $\ln \mu \propto \sqrt{E}$. In the electric field dependence shown in Fig. 8, this behavior is observed, but for larger applied electric fields than in the experimental studies. This discrepancy is expected to be corrected by including a spatial correlation in the diagonal disorder. This is the next obvious step necessary to achieve our goal to successfully model charge transport in conjugated polymers.

\section{ACKNOWLEDGMENTS}

Financial support from the Swedish Research Council (VR) and computational resources and support from the MATTER Network is gratefully acknowledged. M.L. thanks SERC for financial support.

${ }^{1}$ A. Miller and E. Abrahams, "Impurity conduction at low concentrations," Phys. Rev. 120(3), 745-755 (1960).

${ }^{2}$ R. A. Marcus, "On the theory of oxidation-reduction reactions involving electron transfer. I," J. Chem. Phys. 24(5), 966 (1956).

${ }^{3} \mathrm{H}$. Bässler, "Charge transport in disordered organic photoconductors a Monte Carlo simulation study," Phys. Status Solidi B 175(1), 15-56 (1993).

${ }^{4}$ J.-L. Brédas, D. Beljonne, V. Coropceanu, and J. Cornil, "Charge-transfer and energy-transfer processes in pi-conjugated oligomers and polymers: A molecular picture," Chem. Rev. 104(11), 4971-5004 (2004).

${ }^{5}$ V. Lemaur, D. A. da Silva Filho, V. Coropceanu, M. Lehmann, Y. Geerts, J. Piris, M. G. Debije, A. M. van de Craats, K. Senthilkumar, L. D. A. Siebbeles, J. M. Warman, J.-L. Brédas, and J. Cornil, "Charge transport properties in discotic liquid crystals: A quantum-chemical insight into structure-property relationships," J. Am. Chem. Soc. 126(10), 3271-3279 (2004).

${ }^{6}$ Y. Olivier, V. Lemaur, J.-L. Brédas, and J. Cornil, "Charge hopping in organic semiconductors: Influence of molecular parameters on macroscopic mobilities in model one-dimensional stacks," J. Phys. Chem. A 110(19), 6356-6364 (2006)

${ }^{7}$ F. Castet, P. Aurel, A. Fritsch, L. Ducasse, D. Liotard, M. Linares, J. Cornil, and D. Beljonne, "Electronic polarization effects on charge carriers in anthracene: A valence bond study," Phys. Rev. B 77(11), 115210 (2008).
${ }^{8}$ B. J. Schwartz, "Conjugated polymers: What makes a chromophore?," Nature Mater. 7(6), 427-428 (2008).

${ }^{9}$ M. Linares, M. Hultell, and S. Stafström, "The effect of lattice dynamics on electron localization in poly-(para-phenylenevinylene)," Synth. Met. 159(21-22), 2219-2221 (2009).

${ }^{10}$ J. Cornil, D. Beljonne, Z. Shuia, T. W. Hagler, I. Campbell, D. D. C. Bradley, J.-L. Brédas, C. W. Spangler, and K. Müllen, "Vibronic structure in the optical absorption spectra of phenylene vinylene oligomers: A joint experimental and theoretical study," Chem. Phys. Lett. 247(4-6), 425-432 (1995).

${ }^{11}$ J. Cornil, J.-L. Brédas, J. Zaumseil, and H. Sirringhaus, "Ambipolar transport in organic conjugated materials," Adv. Mater. 19(14), 1791-1799 (2007).

${ }^{12}$ L. Martínez, R. Andrade, E. G. Birgin, and J. M. Martínez, "PACKMOL: A package for building initial configurations for molecular dynamics simulations," J. Comput. Chem. 30(13), 2157-64 (2009).

${ }^{13}$ J. M. Martínez and L. Martínez, "Packing optimization for automated generation of complex system's initial configurations for molecular dynamics and docking," J. Comput. Chem. 24(7), 819-25 (2003).

${ }^{14} \mathrm{~W}$. L. Jorgensen and J. Tirado-Rives, "The OPLS [optimized potentials for liquid simulations] potential functions for proteins, energy minimizations for crystals of cyclic peptides and crambin," J. Am. Chem. Soc. 110(6), 1657-1666 (1988).

${ }^{15}$ W. L. Jorgensen, D. S. Maxwell, and J. Tirado-Rives, "Development and testing of the OPLS all-atom force field on conformational energetics and properties of organic liquids," J. Am. Chem. Soc. 118(45), 11225-11236 (1996).

${ }^{16}$ B. Hess, C. Kutzner, D. van der Spoel, and E. Lindahl, "Gromacs 4: Algorithms for highly efficient, load-balanced, and scalable molecular simulation," J. Chem. Theory Comput. 4(3), 435-447 (2008).

${ }^{17}$ D. van der Spoel, E. Lindahl, B. Hess, G. Groenhof, A. E. Mark, and H. J. C. Berendsen, "GROMACs: fast, flexible, and free," J. Comput. Chem. 26(16), 1701-1718 (2005).

${ }^{18}$ E. Lindahl, B. Hess, and D. van der Spoel, "GROMACs 3.0: A package for molecular simulation and trajectory analysis," J. Mol. Model. 7(8), 306317 (2001).

${ }^{19}$ H. J. C. Berendsen, D. van der Spoel, and R. van Drunen, "GROMACs: A message-passing parallel molecular dynamics implementation," Comput. Phys. Commun. 91(1-3), 43-56 (1995).

${ }^{20} \mathrm{~S}$. Nosé, "A molecular dynamics method for simulations in the canonical ensemble," Mol. Phys. 52(2), 255-268 (1984).

${ }^{21}$ W. G. Hoover, "Canonical dynamics: Equilibrium phase-space distributions," Phys. Rev. A 31(3), 1695-1697 (1985).

${ }^{22}$ M. Parrinello and A. Rahman, "Polymorphic transitions in single crystals: A new molecular dynamics method," J. Appl. Phys. 52(12), 7182 (1981).

${ }^{23}$ S. Nosé and M. L. Klein, "Constant pressure molecular dynamics for molecular systems," Mol. Phys. 50(5), 1055-1076 (1983).

${ }^{24} \mathrm{M}$. Gailberger and $\mathrm{H}$. Bässler, "dc and transient photoconductivity of poly(2-phenyl-1,4-phenylenevinylene)," Phys. Rev. B 44(16), 8643-8651 (1991).

${ }^{25}$ M. Jakobsson and S. Stafström, "Polaron effects and electric field dependence of the charge carrier mobility in conjugated polymers," J. Chem. Phys. 135(13), 134902 (2011).

${ }^{26}$ R. S. Mulliken, C. A. Rieke, D. Orloff, and H. Orloff, "Formulas and numerical tables for overlap integrals," J. Chem. Phys. 17(12), 1248 (1949)

${ }^{27}$ A. Hansson and S. Stafström, "Intershell conductance in multiwall carbon nanotubes," Phys. Rev. B 67(7), 075406 (2003).

${ }^{28}$ M. Plato, K. Moebius, M.-E. Michel-Beyerle, M. Bixon, and J. Jortner, "Intermolecular electronic interactions in the primary charge separation in bacterial photosynthesis," J. Am. Chem. Soc. 110(22), 7279-7285 (1988).

${ }^{29}$ L. M. Herz, C. Daniel, C. Silva, F. J. M. Hoeben, A. P. H. J. Schenning, E. W. Meijer, R. H. Friend, and R. T. Phillips, "Fast exciton diffusion in chiral stacks of conjugated p-phenylene vinylene oligomers," Phys. Rev. B 68(4), 045203 (2003)

${ }^{30}$ D. Beljonne, E. Hennebicq, C. Daniel, L. M. Herz, C. Silva, G. D. Scholes, F. J. M. Hoeben, P. Jonkheijm, A. P. H. J. Schenning, S. C. J. Meskers, R. T. Phillips, R. H. Friend, and E. W. Meijer, "Excitation migration along oligophenylenevinylene-based chiral stacks: delocalization effects on transport dynamics," J. Phys. Chem. B 109(21), 10594-10604 (2005).

${ }^{31}$ M. Sing, U. Schwingenschlögl, R. Claessen, P. Blaha, J. Carmelo, L. Martelo, P. Sacramento, M. Dressel, and C. Jacobsen, "Electronic structure 
of the quasi-one-dimensional organic conductor TTF-TCNQ," Phys. Rev. B 68(12), 125111 (2003).

${ }^{32}$ S. Wen, W.-Q. Deng, and K.-L. Han, "Ultra-low resistance at TTF-TCNQ organic interfaces," Chem. Commun. 46(28), 5133-5135 (2010).

${ }^{33}$ H. C. F. Martens, P. W. M. Blom, and H. F. M. Schoo, "Comparative study of hole transport in poly(p-phenylene vinylene) derivatives," Phys. Rev. B 61(11), 7489-7493 (2000).

${ }^{34}$ D. Hertel, H. Bassler, U. Scherf, and H. H. Horhold, "Charge carrier transport in conjugated polymers,” J. Chem. Phys. 110(18), 9214 (1999).
${ }^{35}$ P. W. M. Blom and M. C. J. M. Vissenberg, "Charge transport in poly(pphenylene vinylene) light-emitting diodes," Mater. Sci. Eng. R. 27(3-4), 53-94 (2000).

${ }^{36}$ P. M. Borsenberger, E. H. Magin, M. Van der Auweraer, and F. C. de Schryver, "The role of disorder on charge transport in molecularly doped polymers and related materials," Phys. Status Solidi A 140(1), 9-47 (1993).

${ }^{37}$ E. Lebedev, T. Dittrich, V. Petrova-Koch, S. Karg, and W. Brutting, "Charge carrier mobility in poly(p-phenylenevinylene) studied by the time-of-flight technique,” Appl. Phys. Lett. 71(18), 2686 (1997). 